\title{
Non-singular solution for anisotropic model by gravitational decoupling in the framework of complete geometric deformation (CGD)
}

\author{
S. K. Maurya ${ }^{1, \mathrm{a}}$, Ksh. Newton Singh ${ }^{2, \mathrm{~b}}$, B. Dayanandan ${ }^{1, \mathrm{c}}$ \\ ${ }^{1}$ Department of Mathematical and Physical Sciences, College of Arts and Science, University of Nizwa, Nizwa, Sultanate of Oman \\ ${ }^{2}$ Department of Physics, National Defence Academy, Khadakwasla, Pune 411023, India
}

Received: 1 March 2020 / Accepted: 30 April 2020 / Published online: 19 May 2020

(C) The Author(s) 2020

\begin{abstract}
We presented a non-singular solution of Einstein's field equations using gravitational decoupling by means of complete geometric deformation (CGD) in the anisotropic domain for compact star models. In this approach both the gravitational potentials are deformed as $v=\xi+$ $\beta h(r)$ and $e^{-\lambda}=\mu+\beta f(r)$, where $\beta$ is a coupling constant. Then we solve more complex field equations under above transformations by using a particular form of deformation function $h(r)$ for two different cases namely the mimic constraint for the pressure $\left\{p(r)=\theta_{1}^{1}\right\}$ and the mimic constraint for the density $\left\{\rho(r)=\theta_{0}^{0}\right\}$ (Ovalle in Phys Lett B 788:213, 2019). The compact star models have been constructed by taking $M_{0} / R=0.2$ for two different non-zero values of $\beta$. Moreover, the boundary conditions are also performed for the said complete geometric deformation in the presence of anisotropic matter distribution. We also find pressure, density, anisotropy and causality conditions that are physically acceptable throughout the model. The $M-R$ curve is also presented to support our model for describing a realistic compact object such as neutron stars.
\end{abstract}

\section{Introduction}

In recent days, Ovalle and his collaborators developed a simple, powerful and systematic effective approach the so-called minimal geometric deformation (MGD) approach for decoupling of the gravitational source in general relativity. The minimal geometric deformation (MGD) was initially discovered $[2,3]$ in the framework of Randall-Sundrum brane-word $[4,5]$. Later on, it was extended to derive the new black hole

\footnotetext{
a e-mail: sunil@unizwa.edu.om (corresponding author)

b e-mail: ntnphy@gmail.com

c e-mail: baiju@unizwa.edu.om
}

solutions [6,7]. In this connection, there are several works on MGD with its applications (earlier and recent) can be seen in the following references [8-21]. The decoupling of the gravitational source through MGD is not only a new technique, but it also provides to search the new solutions of Einstein's field equations with numbers of attractive ingredients. This approach has two main characteristics which are mainly as: (A) In order to find a solution of the Einstein's field equations for a more complex energy-momentum tensor $\tilde{T}_{i j}$, we divide this energy-momentum tensor into two or more simpler energy tensor components as,

$\tilde{T}_{i j}=\left\{T_{i j}, \theta_{i j}^{n}\right\}$.

After the splitting of $\tilde{T}_{i j}$, we solve Einstein's equation for each of the above components. In this process, we achieve many solutions corresponding to each above components for the original energy tensor $\tilde{T}_{i j}$. At last, the solution for the Einstein equations corresponding to the original energymomentum tensor $\tilde{T}_{i j}$ can be obtained by a simple linear combination of all the above solutions. (B) while the second procedure is reverse of the above procedure (A), In this approach, we can generalized a simple solution for the Einstein equations into more complex forms. For example to say that we can start through an easy gravitational source corresponding to original energy-momentum tensor $T_{i j}$ and derive a more complex gravitational source by adding an extra energy-momentum tensor,

$T_{i j} \longmapsto \tilde{T}_{i j}=T_{i j}+\theta_{i j}^{1}$.

In this way we can repeat the same procedure by adding more gravitational sources $\theta_{i j}^{n}$ to generalize the solutions of the Einstein equations corresponding to energy tensor $T_{i j}$, into the scenario of more generalize form of the gravitational sources $\tilde{T}_{i j}$. Furthermore, this also implies that they interact only gravitationally. Basically MGD-decoupling was applied 
for a particular transformation which is along the radial metric component $\hat{e}^{-\lambda(r)}$ as: $e^{-\lambda(r)} \mapsto \hat{e}^{-\lambda(r)}=e^{-\lambda(r)}+\beta f(r)$, where $f(r)$ denotes the deformation function along the metric component $\hat{e}^{-\lambda(r)}$. Under this transformation, there are several physically acceptable solutions for spherically symmetric space-time have been derived in the different context [60-62]. This MGD approach is also used to examine the gravitational lensing singularity beyond to the general relativity [63] as well as to discover the critical stability space for Bose-Einstein condensates in gravitational structure [64]. In the presence of variable tension fluid branes, It was also applied to discuss the corrections to dark SU(N) star evident parameters [65]. Based on the above discussion we can say that the minimal geometric deformation decoupling approach is a very powerful technique to discover solutions of Einstein's equation for the self-gravitating stellar objects. But Ovalle and his collaborators proposed that this MGD approach has some limitations as it fails to explain the existence of a stable black hole with a welldefined horizon because of the transformation undergone along only the radial metric component and temporal metric component is unchanged. In this regard, this MGD was extended by deforming of both metric functions, and then obtained modified Schwarzschild geometry, a new solution that describes the brane-world star [6], and derived the corrections to the gravitational wave radiation which is emitted by SU(N) EMGD dark glueball stars mergers [66]. Moreover, the extended gravitational decoupling solution was also obtained in the context of changed matter distribution [21].

In the present article, we have extended MGD by the deforming both gravitational potential functions to obtain a singularity free anisotropic solution for compact objects. The presence of anisotropy inside the stellar model plays an important role to describe more compact object models. Normally the densities of the compact stellar objects are larger than the nuclear matter density. Then it can imagine the presence of the unequal pressures (radial and tangential) that introduce the anisotropy inside the object [22]. In the context of the Newtonian regime, Jeans [23] proposed the first result to assume the anisotropy in self-gravitating stellar objects. Sudden after, Lemaître [24] had also measured the impact of local anisotropy and presented that one can reduce the higher limits forced on the maximum value of the surface gravitational potential in the framework of general relativity (GR). Ruderman [25] provided a remarkable depiction of new realistic self-gravitating objects which indicates that a compact object with matter density $(\rho)$ more than $10^{15} \mathrm{~g} / \mathrm{cm}^{3}$ is probably to be anisotropic, where the nuclear interaction becomes relativistic in nature. Bowers and Liang [26] had shown the effects of the anisotropy on the compact stellar model where they studied the modified hydrostatic equilibrium equation by the inclusion of anisotropy, and then comparative effects of anisotropy on the structure of the static spherically symmetric configurations and with isotropic fluid. For an anisotropic equation of state, Heintzmann and Hillebrandt [27] have examined that the maximum mass of neutron star models at the very high densities lies beyond $3-4 M_{\odot}$. There are several remarkable works on the anisotropic fluid have been done in last several years in different scenario [28-59]. Herrera and Santos [22] studied and discussed almost probable reasons for the presence of local anisotropy in self-gravitating systems with examples of both Newtonian and general relativistic scenario. The article is organized as follows: In Sect. 2, first, we define the modified action $S$ by combining of Einstein-Hilbert action and another extra source through the coupling parameter $\beta$. Then we write the general equation of motion by varying the action $S$ with respect to the metric tensor $g^{i j}$. By using this equation of motion, the explicit form of the Einstein's field equations for spherically symmetric metric has been presented which involves the unknown components of energy-momentum tensor $T_{i j}$ and extra source $\theta_{i j}$ with two unknown metric functions $\lambda(r)$ and $v(r)$. The energy tensor $T_{i j}$ describes the perfect fluid matter distribution. In order to solve the system completely for the anisotropic matter distribution (which is coming due to the presence of extra source $\left.\theta_{i j}\right)$, we apply the extended MGD approach as mentioned in Sect. 3. In this approach we deformed both metric potentials by $v=\xi+\beta h(r)$ and $e^{-\lambda}=\mu+\beta f(r)$. By inserting this transformation in the original system we split this system into two subsystems namely Einstein's system (for perfect fluid matter distribution associated with $T_{i j}$ ) and quasiEinstein system (for the anisotropic source $\theta_{i j}$ ). In Sect. 4, we derived the matching conditions (necessary and sufficient conditions) to determine all the arbitrary constants. The procedures for the finding solution for both systems are given in Sect. 5 as: Initially, we start from known seed isotropic solution, in particular, Tolman IV solution for the Einstein's system (first system) which determines $v(r), \lambda(r)$, isotropic pressure $(p(r))$ and density $\rho(r)$. Then we focus on the second system, quasi-Einstein system' which has five unknown namely two unknown deformation functions $f(r)$ and $h(r)$, and three unknown components for the source $\theta\left(\theta_{0}^{0}, \theta_{1}^{1}\right.$ and $\theta_{2}^{2}$ ). Therefore, we need to specify two additional conditions to close this system. We solve this system by specifying the following conditions: (i) The mimic constraints for the pressure [67] and particular physically motivated ansatz for $h(r)$ as $\theta_{1}^{1}(r)=p(r)$ and $h(r)=\left(r^{2} / A^{2}\right)\left(1+r^{2} / A^{2}\right)$, (ii) The mimic constraints for the density [67] and same particular physically motivated ansatz for $h(r)$ as $\theta_{0}^{0}(r)=\rho(r)$ and $h(r)=\left(r^{2} / A^{2}\right)\left(1+r^{2} / A^{2}\right)$, where $A$ is constant. In this process, we determine other deformation function $f(r)$ and now the system is closed. The physical analysis and concluding remarks are given in Sect. 6 respectively. 


\section{The Einstein field equations for decoupled system}

The modified action for decoupled system can be defined by adding an extra source via coupling parameter $\beta$ as, [1]

$S=S_{E H}+\beta S_{\theta}=\int\left[\frac{R}{16 \pi}+\mathscr{L}_{M}+\beta \mathscr{L}_{\theta}\right] \sqrt{-g} d^{4} x$

where $\mathscr{L}_{M}$ denotes the Lagrangian for matter field while $\mathscr{L}_{\theta}$ is a Lagrangian density for the additional source and the sybmols $R$ and $g$ has their usual meanings. Now the energy momentum tensor $T_{i j}$ for the matter distribution is given by,

$T_{i j}=-\frac{2}{\sqrt{-g}} \frac{\delta\left(\sqrt{-g} \mathscr{L}_{M}\right)}{\delta g^{i j}}$.

It is obviously that the matter Lagrangian $\mathscr{L}_{M}$ depends on only the components of metric tensor $g_{i j}$ and not on their derivatives, so we get the following form of $T_{i j}$,

$T_{i j}=-2 \frac{\partial \mathscr{L}_{M}}{\partial g^{i j}}+g_{i j} \mathscr{L}_{M}$

Similarly, the extra source $\theta_{i j}$ as the energy tensor corresponding to Lagrangian density $\mathscr{L}_{\theta}$ given as,

$\theta_{i j}=-2 \frac{\delta \mathscr{L}_{\theta}}{\delta g^{i j}}+g_{i j} \mathscr{L}_{\theta}$.

After varying the action (3) with respect to the metric tensor $g^{i j}$ we get the following general equations of motion for the decoupled system as,

$R_{i j}-\frac{1}{2} R g_{i j}=-8 \pi T_{i j}^{\mathrm{tot}}$,

with $\quad T_{i j}^{\text {tot }}=T_{i j}+\beta \theta_{i j}$,

where we have chosen the relativistic units $G=c=1$. On the other hand we take,

$T_{i j}=(\rho+p) u_{i} u_{j}-p g_{i j}$,

is the 4-dimensional energy-momentum tensor for the perfect fluid matter distribution with matter density $\rho$, isotropic pressure $p$ and 4-velocity vector field $u^{i}$. The extra source $\theta_{i j}$ in Eq. (8) is coupled in the matter field by the parameter $\beta$ which may contain new fields, such as vector, scalar or tensor fields. This source will create anisotropies in the self-gravitating systems. As we know that the Einstein tensor is always divergence-free. Therefore, the total energy-momentum tensor in Eq. (7) must hold the conservation equation,

$\nabla_{j} T^{(\mathrm{tot}) i j}=0$.

A static spherically symmetric metric in Schwarzschild coordinate is given as,

$d s^{2}=-e^{\lambda(r)} d r^{2}-r^{2}\left(d \theta^{2}+\sin ^{2} \theta d \phi^{2}\right)+e^{\nu(r)} d t^{2}$. where $\lambda(r)$ and $v(r)$ are the functions for the radial vector $r$ only. Then the 4-velocity vector $u^{i}=$ can be defined as: $u^{i}=e^{-v / 2} \delta_{0}^{i}$ within $0 \leq r \leq R$. The Einstein's field equation for decoupled system for the metric (11) can be given as,

$$
\begin{aligned}
& 8 \pi\left(T_{0}^{0}+\beta \theta_{0}^{0}\right)=\mathrm{e}^{-\lambda}\left(\frac{\lambda^{\prime}}{r}-\frac{1}{r^{2}}\right)+\frac{1}{r^{2}}, \\
& 8 \pi\left(T_{1}^{1}+\beta \theta_{1}^{1}\right)=-\mathrm{e}^{-\lambda}\left(\frac{v^{\prime}}{r}+\frac{1}{r^{2}}\right)+\frac{1}{r^{2}}, \\
& 8 \pi\left(T_{2}^{2}+\beta \theta_{2}^{2}\right)=-\frac{\mathrm{e}^{-\lambda}}{4}\left(2 v^{\prime \prime}+v^{\prime 2}+2 \frac{v^{\prime}-\lambda^{\prime}}{r}-v^{\prime} \lambda^{\prime}\right) .
\end{aligned}
$$

where,

$$
T_{0}^{0}=\rho(r), \quad T_{1}^{1}=-p(r), \quad T_{2}^{2}=-p(r),
$$

The linear combination of the Eqs. (12-14), corresponding the conservation Eq. (10), gives (using Eq. (15))

$$
\begin{aligned}
& -\frac{\nu^{\prime}}{2}(\rho+p)-p^{\prime}-\frac{\nu^{\prime} \beta}{2}\left(\theta_{0}^{0}-\theta_{1}^{1}\right)+\beta\left(\theta_{1}^{1}\right)^{\prime} \\
& -\frac{2 \beta}{r}\left(\theta_{2}^{2}-\theta_{1}^{1}\right)=0 .
\end{aligned}
$$

It is noted that we can recover the perfect fluid case for $\beta \rightarrow$ 0 . As we see that the system (12)-(14) having eight unknown functions, namely: two gravitational potentials $\lambda(r)$ (radial metric function) and $v(r)$ (temporal metric function); two physical variables, pressure $p(r)$ and density $\rho(r)$; and three unknown components for $\theta_{i j}$ which implies that the system has infinitely many solutions. Therefore, we need to specify some additional conditions to solve the system (12)-(14). For simplicity, we can describe the effective density as,

$\tilde{\rho}=\rho+\beta \theta_{0}^{0}$,

an effective radial pressure

$\tilde{p}_{r}=p-\beta \theta_{1}^{1}$,

and an effective tangential pressure

$\tilde{p}_{t}=p-\beta \theta_{2}^{2}$.

Then obviously we can clearly define the anisotropy as

$\Pi \equiv \tilde{p}_{t}-\tilde{p}_{r} \equiv \beta\left(\theta_{1}^{1}-\theta_{2}^{2}\right)$.

Now, we are going to discuss a different approach for solving the system.

\section{Gravitational decoupling by complete geometric deformation (CGD)}

In this section, we shall apply the complete geometric deformation to solve the system of Eqs. (12)-(14). In the process, we see that how the gravitational decoupling transform the 
field Eqs. (12)-(14) in two separate systems; namely, the Einstein system associated with the source $T_{i j}$ and quasi-Einstein system for the source $\theta_{i j}$. Let us consider the solution for the eqs. Equations (12)-(14) by taking $\beta=0$ which corresponds to GR perfect fluid solution $\{\mu, \xi, p, \rho\}$. Then the respective line element can be written as,

$d s^{2}=-\mu^{-1} d r^{2}-r^{2}\left(d \theta^{2}+\sin ^{2} \theta d \phi^{2}\right)+e^{\xi(r)} d t^{2}$,

with

$\frac{2 m_{0}(r)}{r} \equiv \frac{8 \pi}{r} \int_{0}^{r} \rho r^{2} d r \equiv 1-\mu$,

is the mass function for the standard GR expression. Now let us move on the coupling parameter $\beta$ to see the impact of the source $\theta_{i j}$ on the perfect fluid solution $\{\mu, \xi, p, \rho\}$. For this purpose, we apply the following transformations in Eq. (21) as $[1,66]$

$$
\begin{aligned}
& \xi \mapsto v=\xi+\beta h(r) \\
& \mu \mapsto e^{-\lambda}=\mu+\beta f(r) .
\end{aligned}
$$

where $h(r)$ and $f(r)$ represent the geometric deformation functions corresponding to the temporal and radial metric components. Moreover, this transformation is the extended case of minimal geometric transformation (MGD), which is called a complete geometric deformation (CGD) or extended geometric deformation along both the radial and temporal components of the line element (21). By plugging the deformed metric functions (15) and (16) in the system of equations (12)-(14), the system divided into two subsystems such as: (i) The first system is described by the Einsteins equations for energy-momentum tensor $T_{\mu \nu}$ as,

$$
\begin{aligned}
& \frac{1}{r^{2}}-\frac{r \mu^{\prime}+\mu}{r^{2}}=8 \pi \rho, \\
& -\frac{1}{r^{2}}+\frac{\mu+r \mu \xi^{\prime}}{r^{2}}=8 \pi p, \\
& \mu\left(\frac{\xi^{\prime \prime}}{2}+\frac{\xi^{\prime 2}}{4}+\frac{\xi^{\prime}}{2 r}\right)+\left(\frac{\xi^{\prime} \mu^{\prime}}{4}+\frac{\mu^{\prime}}{2 r}\right)=8 \pi p .
\end{aligned}
$$

along with conservation equations of (16) for $\beta=0$ gives,

$-\frac{\xi^{\prime}}{2}(\rho+p)-p^{\prime}=0$.

The above Eq. (28) is the linear combination of the Eqs. (25)-(27). The second system corresponding to $\theta_{i j}$ source is known as the quasi-Einstein system, is given by the following field equations,

$$
\begin{aligned}
& -\left(\frac{f^{\prime}}{r}+\frac{f}{r^{2}}\right)=8 \pi \theta_{0}^{0}, \\
& -f\left(\frac{v^{\prime}}{r}+\frac{1}{r^{2}}\right)=8 \pi \theta_{1}^{1}+\frac{\mu h^{\prime}}{r} \\
& -\frac{f}{2}\left(v^{\prime \prime}+\frac{v^{\prime 2}}{2}+\frac{v^{\prime}}{r}\right)-\frac{f^{\prime}}{2}\left(\frac{v^{\prime}}{2}+\frac{1}{r}\right)=8 \pi \theta_{2}^{2}+F_{1},
\end{aligned}
$$

where, the expressions for $F_{1}$ is given as,

$F_{1}=\frac{\mu}{4}\left(2 h^{\prime \prime}+\beta h^{\prime 2}+\frac{2 h^{\prime}}{r}+2 \xi^{\prime} h^{\prime}\right)+\frac{\mu^{\prime} h^{\prime}}{4}$.

The conservation equation for the quasi-Einstein system $\left(\nabla_{j} \theta^{i j}=0\right)$ yields the following equation,

$$
-\frac{\nu^{\prime} \beta}{2}\left(\theta_{0}^{0}-\theta_{1}^{1}\right)+\beta\left(\theta_{1}^{1}\right)^{\prime}-\frac{2 \beta}{r}\left(\theta_{2}^{2}-\theta_{1}^{1}\right)=0 .
$$

From the Eqs. (32) and (33) we conclude that there is no exchange of energy-momentum tensor between the sources $T_{i j}$ and $\theta_{i j}$. Therefore, their interaction is purely gravitational.

\section{Matching conditions for anisotropic stellar model}

The study of the matching condition for the anisotropic matter distribution is a crucial part of the boundary between the geometries for interior $(r<R)$ and exterior $(r>R)$ spacetime. In our present situation, the interior stellar spacetime is given by the extended geometric deformed metric,

$$
\begin{aligned}
d s^{2}= & -\left(1-\frac{2 m(r)}{r}\right)^{-1} d r^{2}-r^{2}\left(d \theta^{2}-\sin ^{2} \theta d \phi^{2}\right) \\
& +e^{\xi(r)} e^{\beta h(r)} d t^{2},
\end{aligned}
$$

where

$$
m(r)=m_{0}(r)-\frac{r \beta}{2} f(r) .
$$

is internal mass of the anisotropic stellar structure corresponding to energy momentum tensor $T_{i j}^{\text {tot }}$, while $m_{0}(r)$ is the mass described in Eq. (22) and deformation functions $f(r)$ and $g(r)$ are still to be determined. Now the inner metric (34) should be smoothly matched with an exterior metric where there is no isotropic fluid matter i.e. isotropic pressure $p^{+}$and density $\rho^{+}$are zero. Since the exterior spacetime $(r>R)$ may contain new fields which are described by $\theta_{i j}$-sector. Therefore, the outer spacetime $(r>R)$ is no more vacuum in the present situation. The general exterior spacetime can be given as,

$d s^{2}=-e^{\lambda^{+}(r)} d r^{2}-r^{2}\left(d \theta^{2}-\sin ^{2} \theta d \phi^{2}\right)+e^{\nu^{+}(r)} d t^{2}$,

where the gravitational potentials $\lambda^{+}(r)$ and $v^{+}(r)$ are determined by exact Schwarzschild solution.

For smooth joining of inner geometry with outer geometry at the boundary of the stellar interior at $r=R$, we apply the well known Israel-Darmois junction conditions $[68,69]$ at $r=R$. These conditions are known as the continuity of the first and second fundamental forms across the boundary $\Sigma$. 
The continuity of the first fundamental form at the boundary of the stellar model $\Sigma$ given as,

$\left[d s^{2}\right]_{\Sigma}=0$

By writing of its explicit form we get,

$$
\begin{aligned}
& \xi(R)+\beta h(R)=v^{+}(R), \\
& 1-\frac{2 M_{0}}{R}+\beta f(R)=e^{-\lambda^{+}(R)},
\end{aligned}
$$

where, $f(R)$ and $h(R)$ are the deformation function at boundary $r=R$ and $M_{0}=m_{0}(R)$. The continuity of the second fundamental can be read as,

$\left[G_{i j} r^{j}\right]_{\Sigma}=0$

here $r_{j}$ is a unit vector. Using Eqs. (7) and (59) we can find

$\left[T_{i j}^{\mathrm{tot}} r^{j}\right]_{\Sigma}=0$

which gives

$\left[p_{r}-\beta \theta_{1}^{1}(r)\right]_{\Sigma}=0$

The condition (42) can be written in the following following final form,

$p_{r}(R)-\beta\left(\theta_{1}^{1}\right)^{-}(R)=-\beta\left(\theta_{1}^{1}\right)^{+}(R)$

where $p_{r}(R)=p_{r}^{-}(R)$. The condition given by Eq. (43) is called the general expression for the second fundamental form connected with the Einstein field equations described by Eq. (7). Now we substitute the value of $\left(\theta_{1}^{1}\right)^{-}(R)$ for the interior geometry from Eq. (32) into the condition (43). Then the second fundamental form (43) can be written as,

$$
\begin{aligned}
& p_{r}(R)+\beta\left[\frac{f}{8 \pi}\left(\frac{v^{\prime}}{r}+\frac{1}{r^{2}}\right)-\frac{\mu h^{\prime}}{r}\right]_{r=R} \\
& =-\beta\left(\theta_{1}^{1}\right)^{+}(R),
\end{aligned}
$$

here $v^{\prime} \equiv \partial_{r} v^{-}$. To obtain $\left(\theta_{1}^{1}\right)^{+}(R)$, we use the Eqs. (30) and (34) for the outer geometry in Eq. (44) which gives,

$$
\begin{aligned}
& p_{r}(R)+\beta\left[\frac{f(R)}{8 \pi}\left(\frac{\nu^{\prime}(R)}{R}+\frac{1}{R^{2}}\right)-\frac{\mu(R) h^{\prime}(R)}{8 \pi R}\right] \\
& =\frac{\beta f^{*}(R)}{8 \pi}\left[\frac{2 M}{R^{3}} \frac{1}{(1-2 M / R)}+\frac{1}{R^{2}}\right] \\
& -\beta \frac{\left[h^{*}(R)\right]^{\prime}}{8 \pi R}\left(1-\frac{2 M}{R}\right),
\end{aligned}
$$

where, the geometric deformation functions $f^{*}(R)$ and $h^{*}(R)$ for the exterior Schwarzschild solution (52) are coming due to the extra source $\theta_{i j}$. It is important remark that if the exterior solution is described only by the Schwarzschild solution (52), then we must have $f^{*}(r)=0$ and $h^{*}(r)=0$. Then the Eq. (45) can be written as,

$$
p_{r}(R)+\beta\left[\frac{f(R)}{8 \pi}\left(\frac{\nu^{\prime}(R)}{R}+\frac{1}{R^{2}}\right)-\frac{\mu(R) h^{\prime}(R)}{8 \pi R}\right]=0 .
$$

The conditions (38), (39) and (46) represent the necessary and sufficient conditions to find the arbitrary constants which are involve in the system.

\section{Anisotropic solution}

In this section, we will solve the Einstein field equations (12)(14) for the anisotropic solution for compact star by using the extended gravitational approach. In this approach we will find the physical variables $\left\{\tilde{p}_{r}, \tilde{p}_{t}, \tilde{\rho}\right\}$, and two unknown functions $v$ and $\lambda$ which are given in the Eqs. (17)-(19) and (11), respectively. The starting point is to find the solution of Einstein's Eqs. (25)-(27) for the perfect distribution. For a particular choice, we choose simply the well-known Tolman IV solution as a seed solution $\{\xi, \mu, p, \rho\}$, given by

$$
\begin{aligned}
& \xi(r)=\ln \left\{\frac{B^{2}}{A^{2}}\left(A^{2}+r^{2}\right)\right\}, \\
& \mu(r)=\frac{\left(C^{2}-r^{2}\right)\left(A^{2}+r^{2}\right)}{C^{2}\left(A^{2}+2 r^{2}\right)}, \\
& p(r)=\frac{C^{2}-A^{2}-3 r^{2}}{8 \pi C^{2}\left(A^{2}+2 r^{2}\right)}, \\
& \rho(r)=\frac{3 A^{4}+A^{2}\left(3 C^{2}+7 r^{2}\right)+2 r^{2}\left(C^{2}+3 r^{2}\right)}{8 \pi C^{2}\left(A^{2}+2 r^{2}\right)^{2}} .
\end{aligned}
$$

where, the arbitrary constants $A, B$ and $C$ will be determined by using the matching conditions. The interior mass $m_{0}(r)$ (as defined in Eq. (22)) is given as,

$m_{0}(r) \equiv 4 \pi \int_{0}^{r} \rho r^{2} d r \equiv \frac{r^{3}\left(A^{2}+C^{2}+r^{2}\right)}{2 C^{2}\left(A^{2}+2 r^{2}\right)}$.

Now let us turn on the second system namely the "quasiEinstein" system corresponding to sector $\theta_{i j}$. Now as we see that the field Eqs. (29)-(31) involves both deformation functions $f(r)$ and $h(r)$ corresponding to radial and temporal metric components, respectively. In order to solve the field Eqs. (29)-(31), we need to specify two more conditions. There are many possibilities like introducing an equation of state (EOS) for the source $\theta_{i j}$ and some physically motivated ansatz for $f(r)$ or $h(r)$ or impose both deformation functions. But in any situation, we must keep in mind that the solution should be physically acceptable. Now we are going to adopt the Ovalle procedures to solve the system (29)-(31) as follows. 
5.1 Solution I: Mimic constraint for the pressure with ansatz for deformation function $h(r)$

In order to close the system (29)-(31), we take the simple choice [67],

$$
\begin{aligned}
& \theta_{1}^{1}(r)=p(r), \\
& h(r)=\frac{r^{2}}{A^{2}}\left(1+\frac{r^{2}}{A^{2}}\right) .
\end{aligned}
$$

The condition (52) is compatible with exterior Schwarzschild solution for a regular solution [67] and ansatz (53) is regular and non-singular throughout within self-gravitating system. Now by plugging the Eqs. (30), (52) and (53), we obtain the deformation function $f(r)$ as,

$$
\begin{aligned}
& f(r)=\frac{r^{2}\left(A^{2}+r^{2}\right)\left[A^{6}-4 C^{2} r^{4}+4 r^{6}+f_{1}(r)\right]}{C^{2}\left(A^{2}+2 r^{2}\right) f_{2}(r)} . \\
& f_{1}(r)=A^{4}\left(-3 C^{2}+5 r^{2}\right)+A^{2}\left(-6 C^{2} r^{2}+6 r^{4}\right), \\
& f_{2}(r)=A^{6}+A^{4}(3+2 \beta) r^{2}+6 A^{2} \beta r^{4}+4 \beta r^{6} .
\end{aligned}
$$

Then the deformed gravitational potentials are given as,

$$
\begin{aligned}
& e^{-\lambda(r)}=\frac{\left(C^{2}-r^{2}\right)\left(A^{2}+r^{2}\right)}{C^{2}\left(A^{2}+2 r^{2}\right)}+\beta f(r), \\
& e^{\nu(r)}=\frac{B^{2}}{A^{2}}\left(A^{2}+r^{2}\right) \cdot \exp \left\{\beta \frac{r^{2}}{A^{2}}\left(1+\frac{r^{2}}{A^{2}}\right)\right\} .
\end{aligned}
$$

and the effective physical quantities $\left\{\tilde{p}_{r}, \tilde{p}_{t}, \tilde{\rho}\right\}$ can be given as,

$$
\begin{aligned}
& \tilde{p}_{r}(r)=p(r)-\beta \theta_{1}^{1}(r)=(1-\beta) \frac{C^{2}-A^{2}-3 r^{2}}{8 \pi C^{2}\left(A^{2}+2 r^{2}\right)}, \\
& \tilde{p}_{t}(r)=p(r)-\beta \theta_{2}^{2}(r)=\frac{C^{2}-A^{2}-3 r^{2}}{8 \pi C^{2}\left(A^{2}+2 r^{2}\right)}-\beta \theta_{2}^{2}(r), \\
& \tilde{\rho}(r)=\frac{3 A^{4}+A^{2}\left(3 C^{2}+7 r^{2}\right)+2 r^{2}\left(C^{2}+3 r^{2}\right)}{8 \pi C^{2}\left(A^{2}+2 r^{2}\right)^{2}} \\
& +\beta \theta_{0}^{0}(r) .
\end{aligned}
$$

where, the expression for $\theta_{0}^{0}(r)$ and $\theta_{2}^{2}(r)$ is given in appendix. The anisotropy for the self-gravitating system can be easily determined by subtracting of Eqs. (57) and (58).

Now using continuity of the first fundamental form which is given by,

$$
\begin{aligned}
& \frac{B^{2}}{A^{2}}\left(A^{2}+R^{2}\right) \cdot \exp \left\{\beta \frac{R^{2}}{A^{2}}\left(1+\frac{R^{2}}{A^{2}}\right)\right\}=1-\frac{2 M}{R}, \\
& \mu(R)+\beta f(R)=1-\frac{2 M_{0}}{R}+\beta f(R)=1-\frac{2 M}{R},
\end{aligned}
$$

where the continuity of the second fundamental form (46) with the mimic constraint (52) leads to

$p(R)-\beta p(R)=0 \Longrightarrow p(R)=0$,

which provides the following expression for constant $A$ as,

$$
C^{2}=A^{2}+3 R^{2} \text {. }
$$

Moreover, the explicit form of the Schwarzschild mass $M$ can be given from Eq.(61) as,

$$
\begin{aligned}
& \frac{M}{R}=\frac{M_{0}}{R} \\
& -\frac{\beta R^{2}\left(A^{2}+R^{2}\right)\left[A^{6}-4 C^{2} R^{4}+4 R^{6}+f_{1}(R)\right]}{2 C^{2}\left(A^{2}+2 R^{2}\right) f_{2}(R)} .
\end{aligned}
$$

On the other hand the constant $B$ can be determined by matching conditions (60) and (61) as,

$$
\begin{aligned}
& \frac{B^{2}}{A^{2}}\left(A^{2}+R^{2}\right) \cdot \exp \left\{\beta \frac{R^{2}}{A^{2}}\left(1+\frac{R^{2}}{A^{2}}\right)\right\} \\
& =1-\frac{2 M_{0}}{R}+\beta f(R),
\end{aligned}
$$

It is very important to determine the effects of the anisotropy on the surface redshift [50]. In our case it can be read as,

$z(R)=\left[1-\frac{2 M(R)}{R}\right]^{-1 / 2}-1$,

while the red-shift inside the stellar model can be calculated by the following formula,

$z(r)=e^{-v(r) / 2}-1$,

where,

$$
\begin{aligned}
& f_{1}(R)=A^{4}\left(-3 C^{2}+5 R^{2}\right)+A^{2}\left(-6 C^{2} R^{2}+6 R^{4}\right), \\
& f_{2}(R)=A^{6}+A^{4}(3+2 \beta) R^{2}+6 A^{2} \beta R^{4}+4 \beta R^{6} .
\end{aligned}
$$

For the solution I, we have chosen the compactness $M_{0} / R=$ 0.2 , which satisfies the Buchdahal limit $4 / 9$, and $A=5.7735$ with different values of coupling parameter $\beta$ to investigate the physical behavior of the solution. From Figs. 1 and 2, we see that the central pressure is decreasing when $\beta$ is decreasing, that happens due to presence of factor $(1-\beta)$ in the effective pressure expression (57) while the densities at center and the surface have opposite behavior than the central pressure (see Table 1). This implies that the coupling parameter $\beta$ plays an important role to decide the matter density within the compact objects. Moreover, the anisotropy is positive and increasing throughout the stellar model that describes a more compact object than the isotropic one (Fig. 3). The causality condition is also satisfied within the stellar model (Fig. 4). The $M-R$ curve is also shown in Fig. 5. From this Fig. 5 one can observe that there mass is increasing with $R$ for $\beta=0$ i.e. in the case of isotropic matter distribution while for $\beta=0.01$ 


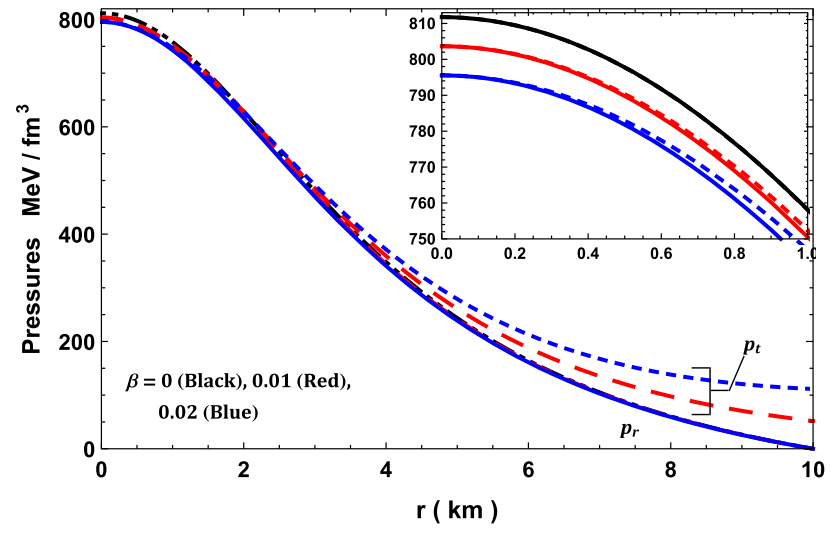

Fig. 1 The trend of thermodynamic pressure against the radial coordinate $r$ for the solution I. For plotting of this figure, the following values have been chosen as $M_{0} / R=0.2, R=10 \mathrm{~km}$ and $A=5.7735$

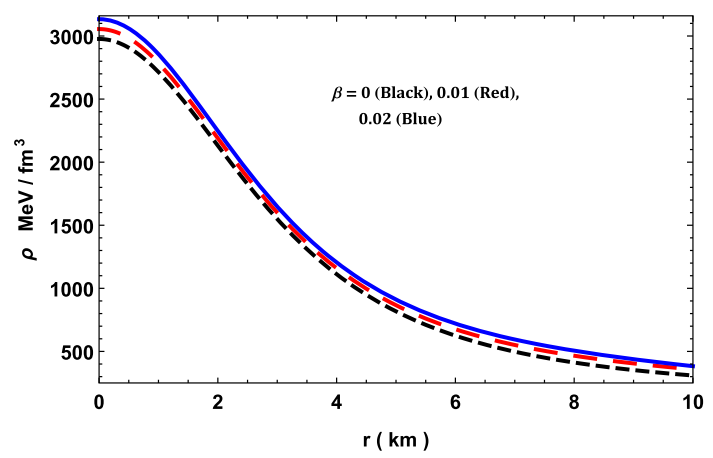

Fig. 2 The trend of energy density against the radial coordinate $r$ for the solution I. For plotting of this figure, the following values have been chosen as $M_{0} / R=0.2, R=10 \mathrm{~km}$ and $A=5.7735$

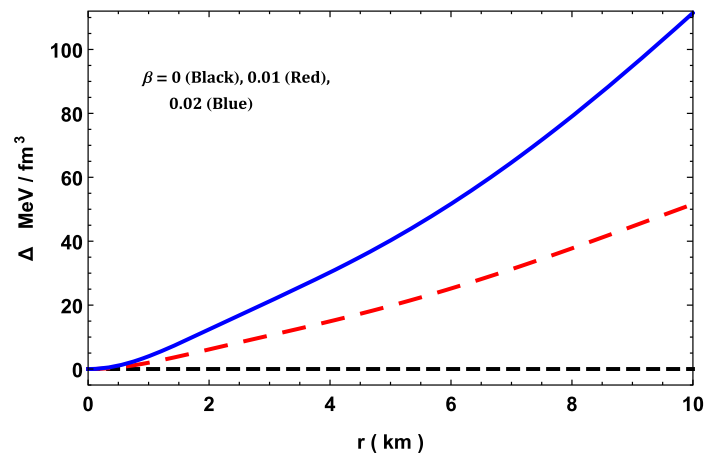

Fig. 3 The trend of anisotropy against the radial coordinate $r$ for the solution I. For plotting of this figure, the following values have been chosen as $M_{0} / R=0.2, R=10 \mathrm{~km}$ and $A=5.7735$

and $\beta=0.002$ the mass and radius are $M_{\max }=1.898$, $R=9.245 M_{\max }$ and $M=1.898, R=10.06 \mathrm{~km}$, respectively. Then corresponding compactness are $M / R=0.3028$ and 0.2783 for $\beta=0.01$ and 0.002 respectively, which also shows that compactness increases when $\beta$ increases. The Fig. 6 shows the trend of gravitational red-shift within the stellar model.

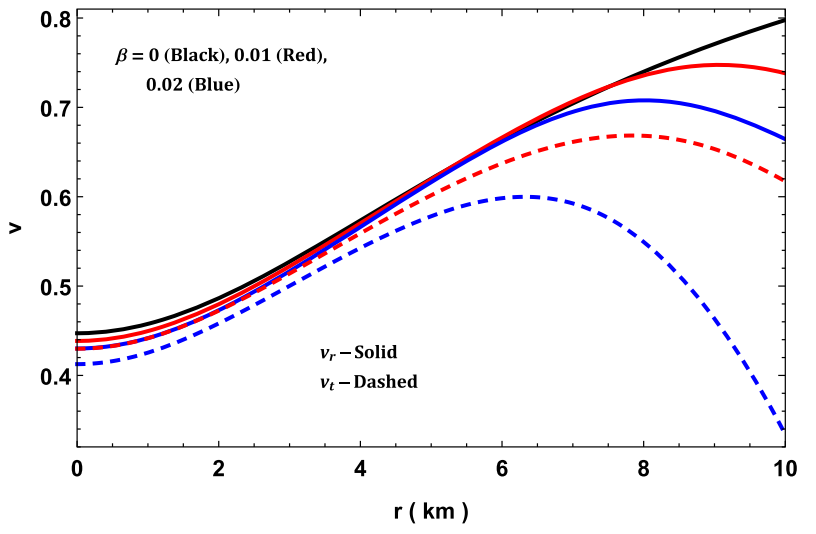

Fig. 4 The trend of velocity of sound against the radial coordinate $r$ for the solution I. For plotting of this figure, the values have been chosen same as Fig. 3

5.2 Solution II: Mimic constraint for the density with same ansatz for deformation function $h(r)$ :

We have adopted another alternative procedure which was proposed by Ovalle [67] as

$$
\theta_{0}^{0}(r)=\rho(r),
$$

This gives the following first order differential equation,

$$
r f^{\prime}(r)+f(r)=-8 \pi r^{2} \rho
$$

which leads the following integral,

$f(r)=-\frac{1}{r} \int 8 \pi r^{2} \rho d r+\frac{C_{1}}{r}$.

For a non singular solution at the center $r=0$, we must put $C_{1}=0$. Then the solution of the Eq. (70) is given as,

$f(r)=-\frac{r^{2}\left(C^{2}+A^{2}+r^{2}\right)}{C^{2}\left(A^{2}+2 r^{2}\right)}$.

Then deformed metric potential is given as,

$$
\begin{aligned}
& e^{-\lambda(r)}=\frac{\left(C^{2}-r^{2}\right)\left(A^{2}+r^{2}\right)}{C^{2}\left(A^{2}+2 r^{2}\right)}-\beta \frac{r^{2}\left(C^{2}+A^{2}+r^{2}\right)}{C^{2}\left(A^{2}+2 r^{2}\right.} \\
& e^{\nu(r)}=\frac{B^{2}}{A^{2}}\left(A^{2}+r^{2}\right) \cdot \exp \left\{\beta \frac{r^{2}}{A^{2}}\left(1+\frac{r^{2}}{A^{2}}\right)\right\}
\end{aligned}
$$

and corresponding physical quantities $\left\{\tilde{p}_{r}, \tilde{p}_{t}, \tilde{\rho}\right\}$ can be written as,

$$
\begin{aligned}
& \tilde{p}_{r}(r)=p(r)-\beta \theta_{1}^{1}(r), \\
& \tilde{p}_{t}(r)=p(r)-\beta \theta_{2}^{2}(r), \\
& \tilde{\rho}(r)=\frac{(1+\beta)\left[3 A^{4}+2 r^{2}\left(C^{2}+3 r^{2}\right)+A^{2}\left(3 C^{2}+7 r^{2}\right)\right]}{8 C^{2} \pi\left(A^{2}+2 r^{2}\right)^{2}}
\end{aligned}
$$


Table 1 Case-I: The numerical values of central pressure, central density, surface density, surface red-shift and constants $A, B$ and $C$ of the different compact objects for different values of $\beta$

\begin{tabular}{llllllll}
\hline$\beta$ & $\begin{array}{l}\text { Central pressure } \\
\tilde{p}_{c}\left(\text { dyne } / \mathrm{cm}^{2}\right)\end{array}$ & $\begin{array}{l}\text { Central density } \\
\tilde{\rho}_{0}\left(\mathrm{gm} / \mathrm{cm}^{3}\right)\end{array}$ & $\begin{array}{l}\text { Surface density } \\
\tilde{\rho}_{s}\left(\mathrm{gm} / \mathrm{cm}^{3}\right)\end{array}$ & $\begin{array}{l}\text { Surface redshift } \\
z_{s}\end{array}$ & $A(\mathrm{~km})$ & $B$ & $C(\mathrm{~km})$ \\
\hline 0.0 & $1.30 \times 10^{35}$ & $5.30 \times 10^{15}$ & $5.45 \times 10^{14}$ & 0.291 & 5.7735 & 0.3198 & 18.2574. \\
0.01 & $1.28 \times 10^{35}$ & $5.45 \times 10^{15}$ & $6.41 \times 10^{14}$ & 0.358 & 5.7735 & 0.3015 & 18.2574 \\
0.02 & $1.27 \times 10^{35}$ & $5.59 \times 10^{15}$ & $6.83 \times 10^{14}$ & 0.415 & 5.7735 & 0.2837 & 18.2574 \\
\hline
\end{tabular}

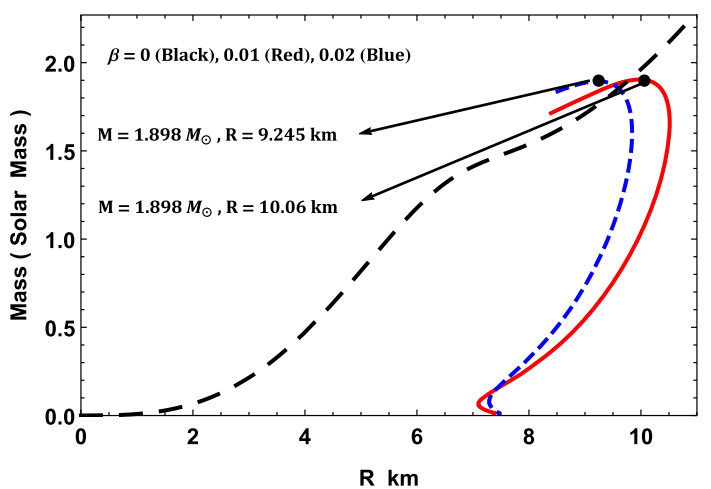

Fig. 5 The $M-R$ curve for solution I

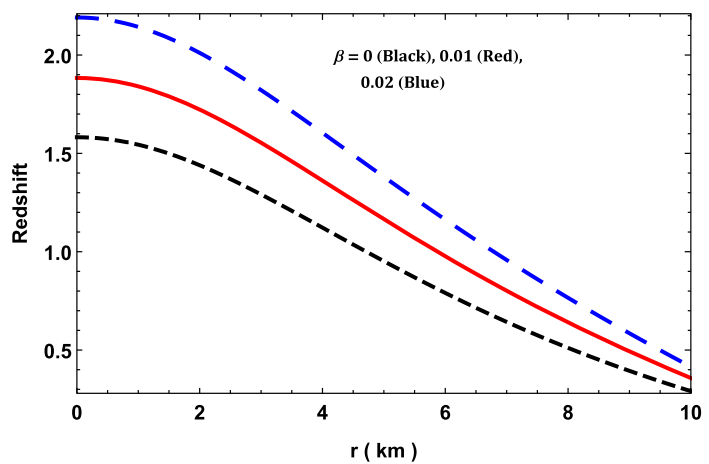

Fig. 6 The trend of redshift against the radial coordinate $r$ for the solution I. For plotting of this figure, the following values have been chosen as $M_{0} / R=0.2, R=10 \mathrm{~km}$ and $A=5.7735$

where, the expression for $\theta_{1}^{1}(r)$ and $\theta_{2}^{2}(r)$ is given in appendix. Now the matching conditions (38) and (39) provides,

$$
\begin{aligned}
& \frac{B^{2}}{A^{2}}\left(A^{2}+R^{2}\right) \cdot \exp \left\{\beta \frac{R^{2}}{A^{2}}\left(1+\frac{R^{2}}{A^{2}}\right)\right\}=1-\frac{2 M}{R}, \\
& \frac{2 M_{0}}{R}-\beta f(R)=\frac{2 M}{R} .
\end{aligned}
$$

where the matching condition (46) can be written as

$p(R)-\beta \theta_{1}^{1}(R)=0$,

which provides the constant $C$ as,

$$
C^{2}=\frac{-\left(A^{2}+R^{2}\right)\left[\Psi_{1}(R)+\Psi_{2}(R)\right]}{A^{6}(1+3 \beta)+A^{4}\left(1+11 \beta+2 \beta^{2}\right) R^{2}+\Psi_{3}(R)},
$$

where, $\Psi_{1}(R)=A^{6}(-1+\beta)+A^{4}\left(-3+\beta+2 \beta^{2}\right) R^{2}$, $\Psi_{2}(R)=6 A^{2}(-1+\beta) b R^{4}+4(-1+\beta) \beta R^{6}, \Psi_{3}(R)=$ $2 A^{2} \beta(5+3 \beta) R^{4}+4 \beta(1+\beta) R^{6}$.

However, the Schwarzschild mass $M$ for this model can be written as (using Eq. (61)),

$$
\frac{M}{R}=\frac{M_{0}}{R}-\frac{\beta R^{2}\left(C^{2}+A^{2}+R^{2}\right)}{2 C^{2}\left(A^{2}+2 R^{2}\right)} .
$$

Using the matching conditions (60) and (61), the constant $B$ can be determined by,

$$
B^{2}\left(1+\frac{R^{2}}{A^{2}}\right)=1-\frac{2 M_{0}}{R}+\beta f(R),
$$

Moereover, surface red-shift and the gravitational red-shift inside the stellar model can be obtained by the same formula (66) and 67, respectively.

For the solution II, we have chosen the same compactness $M_{0} / R=0.2$ and $A=5.7735$ with same two values of coupling parameter $\beta$ to describe the physical behavior of this solution II. From Figs. 7 and 8 , the trend of pressure and density are the same as solution I but effective central pressure and effective density, at center and surface, both having more value than the solution I (see Table 2). In the present case, the anisotropy is also positive and increasing throughout the stellar model which yields a more compact object than isotropic one and has more value than the solution I at the surface (Fig. 9). From Fig. 10, it can clearly be observed that the causality condition holds good throughout the model. We have also plotted the $M-R$ curve for this solution II in Fig. 11. As can see that the maximum mass and radius are $M_{\max }=1.961 M_{\odot}, R=9.685 \mathrm{~km}$, $M_{\max }=2.012 M_{\odot}, R=9.72 \mathrm{~km}$, and $M_{\max }=2.062 M_{\odot}$, $R=9.799 \mathrm{~km}$ respectively for $\beta=0.0,0.01$ and 0.002 . Moreover, the compactness are $M / R=0.29865,0.30532$, 0.31038 for $\beta=0.0,0.01,0.02$, respectively. We note that the shape of $M-R$ curves describes the similar shape of the neutron star models. The variation of the gravitational redshift can be seen in Fig. 12. 


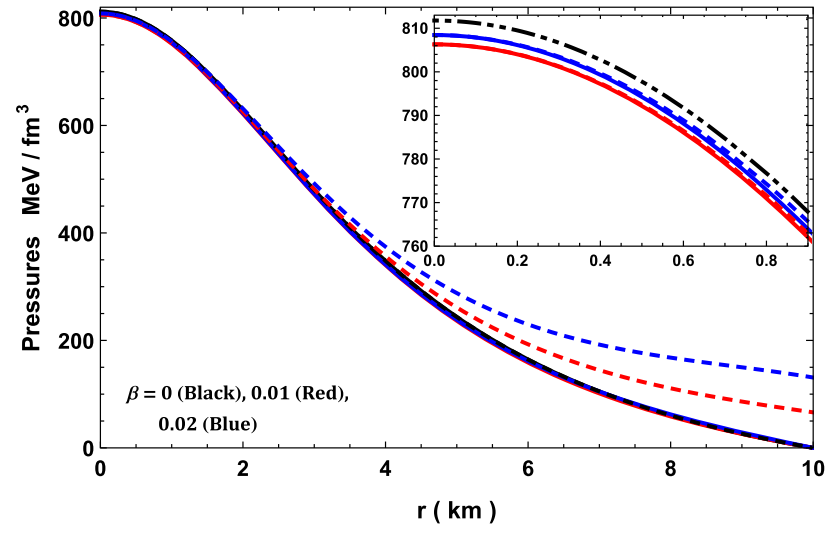

Fig. 7 The trend of thermodynamic pressures against the radialcoordinate $r$ for the solution II. For plotting of this figure, the following values have been chosen as $M_{0} / R=0.2, R=10 \mathrm{~km}$ and $A=5.7735$

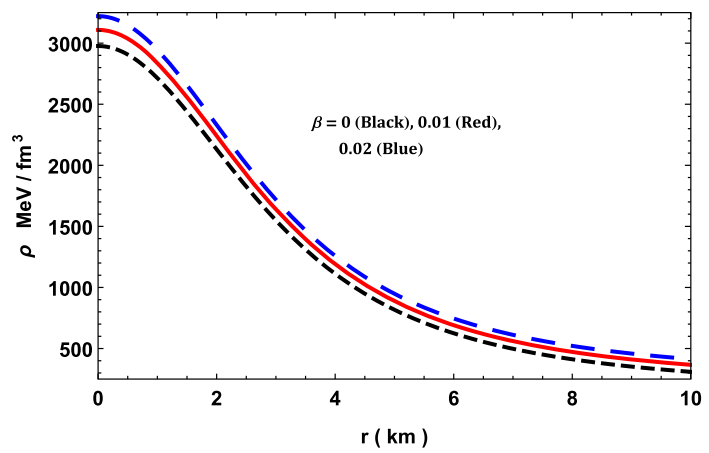

Fig. 8 The trend of energy density against the radial coordinate $r$ for the solution II. For plotting of this figure, the values are been chosen same as Fig. 7

\section{Conclusion}

In the present article, we have explained the gravitational decoupling approach in the framework of complete geometric (CGD) to investigate or generalize the interior isotropic solution for a self-gravitating system in the presence of anis- otropic gravitational source. In order to describe this approach, first we have defined the Einstein field equations (12)-(14) of the effective energy-momentum tensor $\tilde{T}_{i j}$, which is combination of energy-momentum tensor $T_{i j}$ and extra source $\theta_{i j}$, for spherically symmetric metric in terms of two unknown gravitational potential $e^{\nu(r)}$ and $e^{\lambda(r)}$. The

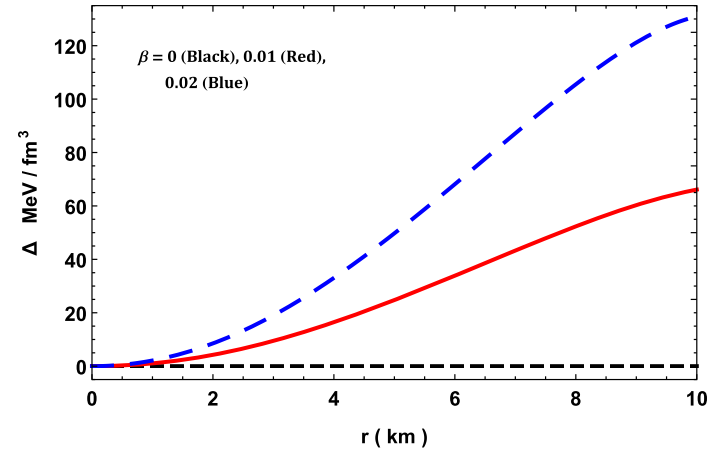

Fig. 9 The trend of anisotropy against the radial coordinate $r$ for the solution II. For plotting of this figure, the values have been chosen same as Fig. 7

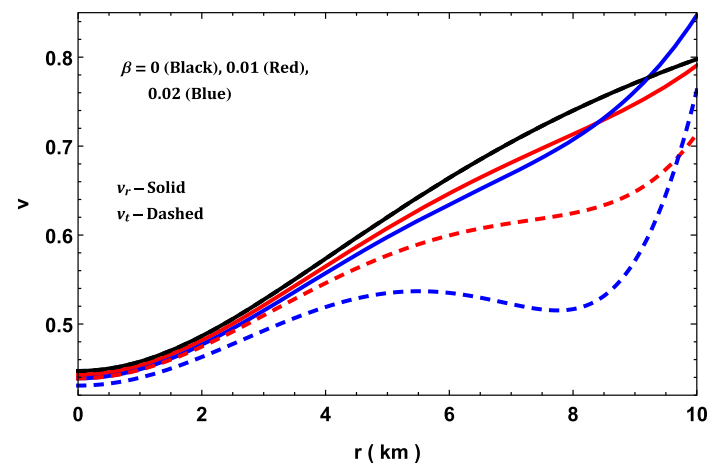

Fig. 10 The trend of velocity of sound against the radial coordinate $r$ for the solution II. For plotting of this figure, the following values have been chosen as $M_{0} / R=0.2, R=10 \mathrm{~km}$ and $A=5.7735$

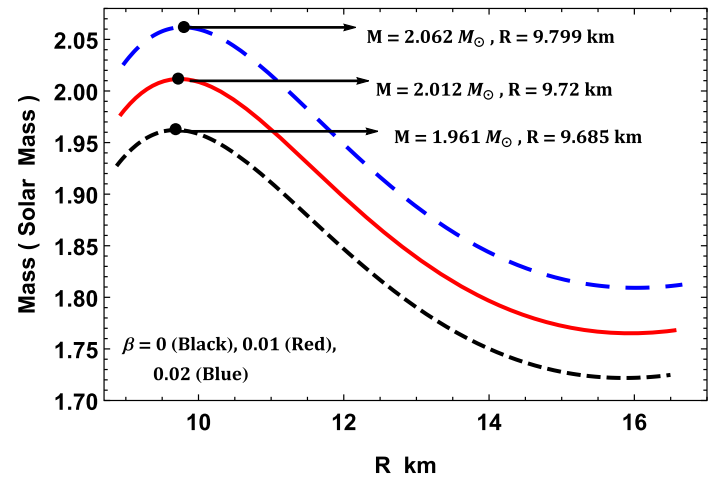

Fig. 11 The $M-R$ curve for Solution II

Table 2 Case-II: The numerical values of central pressure, central density, surface density, surface red-shift and constants $A, B$ and $C$ of the different compact objects for different values of $\beta$

\begin{tabular}{llllllll}
\hline$\beta$ & $\begin{array}{l}\text { Central pressure } \\
\tilde{p}_{c}\left(\text { dyne } / \mathrm{cm}^{2}\right)\end{array}$ & $\begin{array}{l}\text { Central density } \\
\tilde{\rho}_{0}\left(\mathrm{gm} / \mathrm{cm}^{3}\right)\end{array}$ & $\begin{array}{l}\text { Surface density } \\
\tilde{\rho}_{s}\left(\mathrm{gm} / \mathrm{cm}^{3}\right)\end{array}$ & $\begin{array}{l}\text { Surface redshift } \\
z_{s}\end{array}$ & $A(\mathrm{~km})$ & $B$ & $C(\mathrm{~km})$ \\
\hline 0.0 & $1.301 \times 10^{35}$ & $5.29 \times 10^{15}$ & $5.48 \times 10^{14}$ & 0.291 & 5.7735 & 0.3198 & 18.2574. \\
0.01 & $1.295 \times 10^{35}$ & $5.52 \times 10^{15}$ & $6.47 \times 10^{14}$ & 0.284 & 5.7735 & 0.3015 & 17.8849 \\
0.02 & $1.292 \times 10^{35}$ & $5.74 \times 10^{15}$ & $7.33 \times 10^{14}$ & 0.276 & 5.7735 & 0.2837 & 17.5437 \\
\hline
\end{tabular}




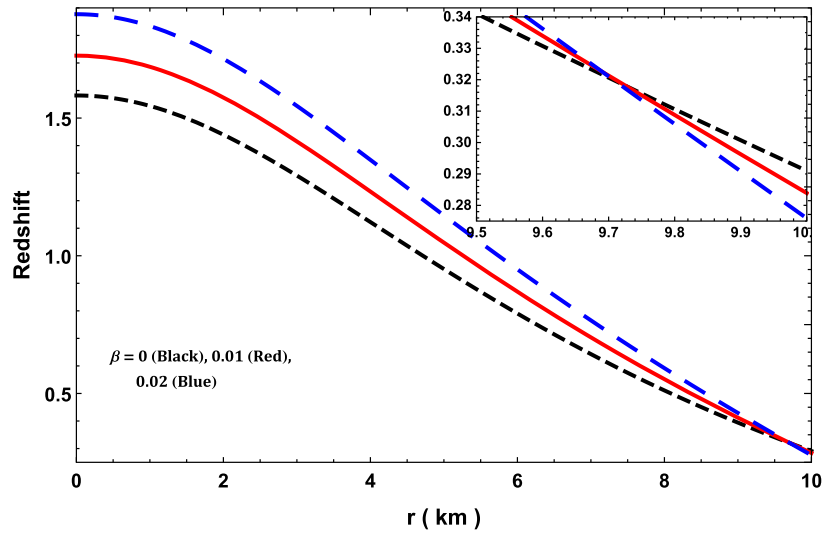

Fig. 12 The trend of redshift against the radial coordinate $r$ for the solution II. For plotting of this figure, the following values have been chosen as $M_{0} / R=0.2, R=10 \mathrm{~km}$ and $A=5.7735$

source $T_{i j}$ associated with the isotropic sector (9) means the perfect fluid matter distribution while the source $\theta_{i j}$ introduces the anisotropy in the self-gravitating system. Next we apply the Ovalle [1] transformations, known as extended geometric deformation, $v=\xi+\beta h(r)$ and $e^{-\lambda}=\mu+\beta f(r)$ and split the original system (12)-(14) into two sub-system namely Einstein system for perfect fluid distributions (25)(27) and quasi-Einstein Einstein system for the anisotropic source $\theta_{i j}$ (29)-(31). Moreover, we would like to mention that these two source acts only gravitationally and there is no exchange of the energy-momentum.

We have also performed the matching conditions at the boundary of the stellar system in detail for an exterior Schwarzschild spacetime. Here we have derived an important condition especially the second fundamental form (40) which yields zero effective radial pressure on the surface of the stellar model i.e. $\tilde{p}_{r}=0$ at $r=R$. It is note that the effective radial pressure $\left(\tilde{p}_{r}\right)$ contains the isotropic pressure $(p)$, when $\beta=0$, and both inner geometric deformation functions $f(r)$ and $h(r)$ due to extra source $\theta_{i j}$. From the Eq. (46), it is clear that the exterior Schwarzschild solution is compatible with the non-vanishing source $\theta_{i j}$ if the isotropic pressure is negative at the boundary of the stellar model i.e. $p(R)<0$. In order to test this methodology in the context of physical acceptability, we have taken Tolman IV solution as a seed solution for the system (25)-(27) which yields directly $\mu(r)$ and $\nu(r)$. To close the quasi-Einstein system we have specified a particular form of deformation function $h(r)=\left(r^{2} / A^{2}\right)\left(1+r^{2} / A^{2}\right)$ and two different conditions namely (i) The mimic constraints for the pressure i.e. $\theta_{1}^{1}(r)=p(r)$, and (ii) The mimic constraints for the density i.e. $\theta_{0}^{0}(r)=\rho(r)$. We have solved the quasiEinstein's system for both conditions and obtained the solution for effective energy-momentum tensor $\tilde{T}_{i j}$ by combing the solutions of both systems. We have constructed the selfgravitating compact objects by taking particular values of the coupling parameter $\beta$. This because for $\beta>0.02$, the transverse sound speed near the surface becomes imaginary. Also, here we choose the same values of some parameters such as $M, R, A$ and $M_{0} / R$ in both the solutions so that we can compare their behaviour and nature of equation of states. One can be seen that the nature of energy density, pressures, redshift and anisotropy are almost the same however, the trends of sound velocity is different. Although, the anisotropy is more for solution II as compared to I and in contrast, the central redshift is more for solution I than II. From Figs. 4 and 10 we can see that the velocity of sound is also more for solution II than I implying that the solution II has stiffer equation of state than I. As a consequence the $M_{\max }$ is more for solution II than I. Interestingly, the $M_{\max }$ yielded from solution I is independent of the coupling parameter $\beta$. However, this is not the same for solution II as one can see that when the coupling strength $(\beta)$ increases the $M_{\max }$ increases. Therefore, it can be concluded that solution I's Eos has no effect from the coupling strength while for solution II the stiffness of the EoS increases with coupling strength.

Finally, we emphasize that the minimal geometric deformation (MGD) is not just a tool for exploring new physically reliable solution for anisotropic matter distribution, but gives a strong and effective approach to introduce the gravitational decoupling in concern physical problems. However, the extension of this MGD i.e. deformation over both gravitational potential, or complete geometric deformation provides a more complex system of equations. The solution to this complex system is not a trivial task. Only a few solutions are available in the literature. Therefore, still we need to discover an effective way by using gravitational decoupling in the framework of CGD to solve this complex system for different matter or scalar fields.

Acknowledgements S. K. Maurya acknowledge that this work is carried out under the TRC project-BFP/RGP/CBS/19/099 of the Sultanate of Oman.

Data Availability Statement This manuscript has no associated data or the data will not be deposited. [Authors' comment: This is a theoretical investigations where the authors have generated all the graphs analytically using Mathematica.]

Open Access This article is licensed under a Creative Commons Attribution 4.0 International License, which permits use, sharing, adaptation, distribution and reproduction in any medium or format, as long as you give appropriate credit to the original author(s) and the source, provide a link to the Creative Commons licence, and indicate if changes were made. The images or other third party material in this article are included in the article's Creative Commons licence, unless indicated otherwise in a credit line to the material. If material is not included in the article's Creative Commons licence and your intended use is not permitted by statutory regulation or exceeds the permitted use, you will need to obtain permission directly from the copyright holder. To view a copy of this licence, visit http://creativecomm ons.org/licenses/by/4.0/.

Funded by $\mathrm{SCOAP}^{3}$. 


\section{Appendix}

Solution-I: The mimic constraint for the pressure $\theta_{1}^{1}(r)=p(r)$

$$
\begin{aligned}
\theta_{0}^{0}(r)=\{ & -3 A^{16}+16 A^{2} \beta r^{12}\left(7 C^{2}-25 r^{2}\right) \\
& +32 \beta r^{14}\left(C^{2}-3 r^{2}\right)+A^{14}\left[9 C^{2}-(35+2 \beta) r^{2}\right] \\
& +24 A^{4} r^{10}\left[(5+6 \beta) C^{2}-7(1+4 \beta) r^{2}\right] \\
& +A^{12} r^{2}\left[6(10+\beta) C^{2}-(161+26 \beta) r^{2}\right] \\
& +A^{10} r^{4}\left[(187+24 \beta) C^{2}-(401+122 \beta) r^{2}\right] \\
& +4 A^{6} r^{8}\left[(80+23 \beta) C^{2}-(120+151 \beta) r^{2}\right] \\
& \left.+2 A^{8} r^{6}\left[(170+23 \beta) C^{2}-(296+167 \beta) r^{2}\right]\right\} / \\
& +\left[8 \pi C ^ { 2 } ( A ^ { 2 } + 2 r ^ { 2 } ) ^ { 2 } \left(A^{6}+A^{4}(3+2 \beta) r^{2}\right.\right. \\
& \left.\left.+6 A^{2} \beta r^{4}+4 \beta r^{6}\right)^{2}\right], \\
\theta_{2}^{2}(r)=- & \left\{A^{24}+384 A^{2} \beta^{3} r^{20}\left(C^{2}-r^{2}\right)\right. \\
& +64 \beta^{3} r^{22}\left(C^{2}-r^{2}\right)+64 A^{4} \beta^{2} r^{18}\left[(2+15 \beta) C^{2}\right. \\
& \left.-2(1+7 \beta) r^{2}\right]+A^{22}\left[-C^{2}+(11+3 \beta) r^{2}\right] \\
& +16 A^{6} \beta^{2} r^{16}\left[(40+81 \beta) C^{2}-(40+61 \beta) r^{2}\right] \\
& +A^{20} r^{2}\left[(1+2 \beta) C^{2}+2\left(20+18 \beta+3 \beta^{2}\right) r^{2}\right] \\
& +4 A^{10} \beta r^{12}\left[\left(121+324 \beta+117 \beta^{2}\right) C^{2}\right. \\
& \left.+3\left(-37-54 \beta+25 \beta^{2}\right) r^{2}\right] \\
& +4 A^{8} \beta r^{14}\left[\left(31+318 \beta+255 \beta^{2}\right) C^{2}\right. \\
& \left.-\left(29+276 \beta+87 \beta^{2}\right) r^{2}\right] \\
& +A^{18} r^{4}\left[\left(26+37 \beta+2 \beta^{2}\right) C^{2}\right. \\
& \left.+\left(51+141 \beta+70 \beta^{2}+4 \beta^{3}\right) r^{2}\right] \\
& +A^{16} r^{6}\left[\left(77+201 \beta+34 \beta^{2}\right) C^{2}\right. \\
& \left.-\left(13-191 \beta-302 \beta^{2}-44 \beta^{3}\right) r^{2}\right] \\
& +A^{14} r^{8}\left[\left(91+525 \beta+222 \beta^{2}+12 \beta^{3}\right) C^{2}\right. \\
& \left.+2\left(-34-52 \beta+285 \beta^{2}+96 \beta^{3}\right) r^{2}\right] \\
& +A^{12} r^{10}\left[\left(36+719 \beta+726 \beta^{2}\right.\right. \\
& \left.\left.\left.+116 \beta^{3}\right) C^{2}-\left(30+515 \beta-276 \beta^{2}-400 \beta^{3}\right) r^{2}\right]\right\} \\
& \times\left[8 \pi A ^ { 8 } C ^ { 2 } ( A ^ { 4 } + 3 A ^ { 2 } r ^ { 2 } + 2 r ^ { 4 } ) \left(A^{6}+A^{4}(3+2 \beta) r^{2}\right.\right. \\
& \left.\left.+6 \beta A^{2} r^{4}+4 \beta r^{6}\right)^{2}\right]^{-1} . \\
&
\end{aligned}
$$

Solution-II: The mimic constraint for the density $\theta_{0}^{0}(r)=\rho(r)$

$$
\begin{aligned}
\theta_{1}^{1}(r)=- & \left\{A^{8}+4 r^{6}\left[(1+\beta) C^{2}+(-1+\beta) r^{2}\right]\right. \\
& +2 A^{2} r^{4}\left[(5+3 \beta) C^{2}+5(-1+\beta) r^{2}\right] \\
& +A^{6}\left[3 C^{2}+2(1+\beta) r^{2}\right]+A^{4} r^{2}\left[(11+2 \beta) C^{2}\right. \\
& \left.\left.+(-5+8 \beta) r^{2}\right]\right) /\left[8 \pi A^{4} C^{2}\left(A^{4}+3 A^{2} r^{2}+2 r^{4}\right)\right], \\
\theta_{2}^{2}(r)=- & \left\{A^{12}+4 \beta r^{10}\left[(3+2 \beta) C^{2}+(-3+2 \beta) r^{2}\right]\right. \\
& +4 A^{2} \beta r^{8}\left[(9+4 \beta) C^{2}+3(-3+2 \beta) r^{2}\right] \\
& +A^{10}\left[3 C^{2}+(1+3 \beta) r^{2}\right]+A^{8} r^{2}\left[3(5+2 \beta) C^{2}\right.
\end{aligned}
$$

$$
\begin{aligned}
& \left.-\left(13-16 \beta-2 \beta^{2}\right) r^{2}\right]+A^{6} r^{4} \\
& \times\left[\left(23+33 \beta+2 \beta^{2}\right) C^{2}+4\left(-7+4 \beta+3 \beta^{2}\right) r^{2}\right] \\
& \left.+A^{4} r^{6}\left[\left(12+55 \beta+10 \beta^{2}\right) C^{2}\left(14+21 \beta-26 \beta^{2}\right) r^{2}\right]\right\} \\
& /\left[8 \pi A^{8} C^{2}\left(A^{4}+3 A^{2} r^{2}+2 r^{4}\right)\right] .
\end{aligned}
$$

\section{References}

1. J. Ovalle, Phys. Lett. B 788, 213 (2019)

2. J. Ovalle, Mod. Phys. Lett. A 23, 3247 (2008)

3. J. Ovalle, Gravitation and Astrophysics (ICGA9), Ed. J. Luo, World Scientific, Singapore, 173-182 (2010)

4. L. Randall, R. Sundrum, Phys. Rev. Lett. 83, 3370 (1999)

5. L. Randall, R. Sundrum, Phys. Rev. Lett. 83, 4690 (1999)

6. R. Casadio, J. Ovalle, R. da Rocha, Class. Quant. Gravit. 32, 215020 (2015)

7. J. Ovalle, Int. J. Mod. Phys. Conf. Ser. 41, 1660132 (2016)

8. R. Casadio, J. Ovalle, Phys. Lett. B 715, 251 (2012)

9. J. Ovalle, F. Linares, Phys. Rev. D 88, 104026 (2013)

10. J. Ovalle, F. Linares, A. Pasqua, A. Sotomayor, Class. Quant. Gravit. 30, 175019 (2013)

11. R. Casadio, J. Ovalle, R. da Rocha, Class. Quant. Gravit. 30, 175019 (2014)

12. R. Casadio, J. Ovalle, R. da Rocha, Europhys. Lett. 110, 40003 (2015)

13. J. Ovalle, L.A. Gergely, R. Casadio, Class. Quant. Gravit. 32, 045015 (2015)

14. R. da Rocha, Eur. Phys. J. C 77, 355 (2017)

15. E. Morales, F. Tello-Ortiz, Eur. Phys. J. C 78, 841 (2018)

16. M. Estrada, F. Tello-Ortiz, Eur. Phys. J. Plus 133, 453 (2018)

17. L. Gabbanelli, A. Rincón, C. Rubio, Eur. Phys. J. C 78, 370 (2018)

18. E. Morales, F. Tello-Ortiz, Eur. Phys. J. C 78, 618 (2018)

19. J. Ovalle, R. Casadio, R. da Rocha, A. Sotomayor, Z. Stuchlik, Eur. Phys. J. C 78, 960 (2018)

20. E. Contreras, P. Bargueño, Eur. Phys. J. C 78, 558 (2018)

21. S.K. Maurya, Eur. Phys. J. C 79, 958 (2019)

22. L. Herrera, N.O. Santos, Phys. Rep. 286, 53 (1997)

23. J.H. Jeans, Mon. Not. R. Astron. Soc. 82, 122 (1922)

24. G. Lemaitre, Ann. Soc. Sci. Bruxelles A 53, 51 (1933)

25. R. Ruderman, Rev. Astron. Astrophys. 10, 427 (1972)

26. R.L. Bowers, E.P.T. Liang, Astrophys. J. 188, 657 (1974)

27. H. Heintzmann, W. Hillebrandt, Astron. Astrophys. 38, 51 (1975)

28. L. Herrera, J. Ponce de Leon, J. Math. Phys. 26, 2302 (1985)

29. L. Herrera, Phys. Lett. A 165, 206 (1992)

30. R. Chan, L. Herrera, N.O. Santos, Mon. Not. R. Astron. Soc. 265, 533 (1993)

31. L. Herrera, J. Ospino, A.D. Prisco, Phys. Rev. D 77, 027502 (2008)

32. T. Harko, M.K. Mak, Chin. J. Astron. Astrophys. 2, 248 (2002)

33. T. Harko, M.K. Mak, Ann. Phys. (Amsterdam) 11, 3 (2002)

34. M.K. Mak, N. Dobson Jr., T. Harko, Int. J. Mod. Phys. D 11, 207 (2002)

35. M.K. Mak, T. Harko, Proc. R. Soc. A 459, 393 (2003)

36. T. Harko, M.K. Mak, Class. Quant. Gravity 21, 1489 (2004)

37. C.M. Chaisi, S.D. Maharaj, Gen. Relat. Gravity. 37, 1177 (2005)

38. S. Thirukkanesh, S.D. Maharaj, Class. Quant. Gravity 25, 235001 (2008)

39. J.M. Sunzu, S.D. Maharaj, S. Ray, Astrophys. Space Sci. 352, 719 (2014)

40. S.K. Maurya, Y.K. Gupta, S. Ray, B. Dayanandan, Eur. Phys. J. C 75, 225 (2015)

41. B.S. Ratanpal, V.O. Thomas, D.M. Pandya, Astrophys. Space Sci. 361, 65 (2016)

42. P.M. Takisa, S.D. Maharaj, Astrophys. Space Sci. 361, 262 (2016) 
43. S.K. Maurya, Y.K. Gupta, Astrophys. Space Sci. 344, 243 (2013)

44. K.N. Singh, N. Pant, N. Pradhan, Astrophys. Space Sci. 361, 173 (2016)

45. S.K. Maurya, Y.K. Gupta, S. Ray, D. Deb, Eur. Phys. J. C 76, 693 (2016)

46. S.K. Maurya, Y.K. Gupta, T.T. Smitha, F. Rahaman, Eur. Phys. J. A 52, 191 (2016)

47. S.K. Maurya, Y.K. Gupta, S. Ray, Eur. Phys. J. C 77, 360 (2017)

48. S.K. Maurya, S.D. Maharaj, Eur. Phys. J. C 77, 328 (2017)

49. S.K. Maurya, Y.K. Gupta, B. Dayanandan, M.K. Jasim, A. AlJamel, Int. J. Mod. Phys. D 26, 1750002 (2017)

50. K.N. Singh, N. Pant, M. Govender, Eur. Phys. J. C 77, 100 (2017)

51. S.K. Maurya, A. Banerjee, S. Hansraj, Phys. Rev. D 97, 044022 (2018)

52. S.K. Maurya, A. Banerjee, Y.K. Gupta, Astrophys. Space Sci. 363, $208(2018)$

53. M.K. Jasim, D. Deb, S. Ray, Y.K. Gupta, S.R. Chowdhury, Eur. Phys. J. C 78, 603 (2018)

54. S.K. Maurya, S.D. Maharaj, J. Kumar, A.K. Prasad, Gen. Relat. Gravit 51, 86 (2019)

55. D. Deb, S.V. Ketov, S.K. Maurya, M. Khlopov, P.H.R.S. Moraes, S. Ray, Mon. Not. R. Astron. Soc. 485, 5652 (2019)

56. S.K. Maurya, A. Banerjee, M.K. Jasim, J. Kumar, A.K. Prasad, A. Pradhan, Phys. Rev. D 99, 044029 (2019)
57. S.K. Maurya, A. Errehymy, D. Deb, F. Tello-Ortiz, M. Daoud, Phys. Rev. D 100, 044014 (2019)

58. S.K. Maurya, A. Banerjee, F. Tello-Ortiz, Phys. Dark Univ. 27, $100438(2020)$

59. S.K. Maurya, F. Tello-Ortiz, Ann. Phys. 414, 168070 (2020)

60. S.K. Maurya, F. Tello-Ortiz, Eur. Phys. J. C 79, 85 (2019)

61. K.N. Singh, S.K. Maurya, M.K. Jasim, F. Rahaman, Eur. Phys. J. C 79, 851 (2019)

62. S.K. Maurya, F. Tello-Ortiz, Phys. Dark Univers. 27, 100442 (2020)

63. R.T. Cavalcanti, A. Goncalves da Silva, R. da Rocha, Class. Quant. Gravit. 33, 215007 (2016)

64. R. Casadio, R. da Rocha, Phys. Lett. B 763, 434 (2016)

65. R. da Rocha, Phys. Rev. D 95, 124017 (2017)

66. A. Fernandes-Silva, A.J. Ferreira-Martins, R. da Rocha, Eur. Phys. J. C 78, 631 (2018)

67. J. Ovalle, R. Casadio, R. da Rocha, A. Sotomayor, Eur. Phys. J. C 78, $122(2018)$

68. W. Israel, Nuovo Cimento B 44, 1 (1966)

69. G. Darmois, Memorial des Sciences Mathematiques (GauthierVillars, Paris, 1927) 